\title{
Erratum
}

\section{Vol. 66, No. 14}

On page 392 in "QuickStats: Percentage Distribution of Gestational Age in Weeks for Infants Who Survived to Age 1 Year and Infants Who Died Before Age 1 Year — National Vital Statistics System, United States, 2014," the second and third sentences of the caption should have read as follows: "In $2014,66 \%$ of infants who survived to age 1 year were delivered at full term or later ( $\geq 39$ completed weeks) compared with $19 \%$ of infants who died before reaching age 1 year. Fifty-four percent of infants who died before age 1 year were delivered at $<32$ weeks gestation compared with only $1 \%$ of infants who survived to age 1 year." 